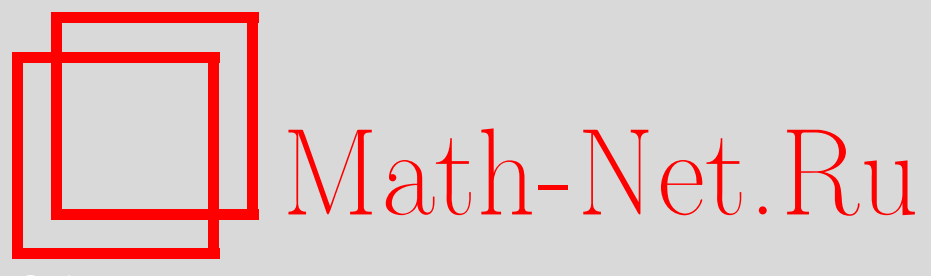

М. В. Шамолин, Случай полной интегрируемости в динамике четырехмерного твердого тела в неконсервативном поле, УМН, 2010, том 65, выпуск 1, 189-190

DOI: https://doi.org/10.4213/rm9320

Использование Общероссийского математического портала Math-Net.Ru подразумевает, что вы прочитали и согласны с пользовательским соглашением http: //www. mathnet.ru/rus/agreement

Параметры загрузки:

IP: 107.22 .136 .117

26 апреля 2023 г., 15:50:45

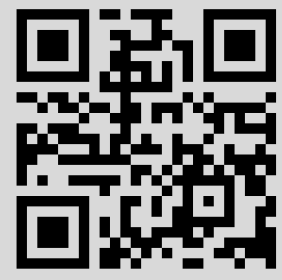




\section{Случай полной интегрируемости в динамике четырехмерного твердого тела в неконсервативном поле}

\section{М. В. Шамолин}

Статья продолжает исследование по поиску новых интегрируемых случаев в динамике четырехмерного твердого тела на $\mathbb{R}^{4} \times \operatorname{so}(4)$ в неконсервативном поле сил [1]-[3]. Ранее автором был предъявлен случай полной интегрируемости уравнений движения динамически симметричного тела при выполнении равенства $I_{1} \neq I_{2}=I_{3}=I_{4}$ [1]. В данной же работе полностью разобран случай другой логически возможной динамической симметрии.

Пусть четырехмерное твердое тело $\Xi$ с гладкой границей $\partial \Xi$ является динамически симметричным (в некоторой системе координат $D x_{1} x_{2} x_{3} x_{4}$ оператор инерции имеет вид $\left.\operatorname{diag}\left\{I_{1}, I_{1}, I_{3}, I_{3}\right\}\right)$. Таким образом, двумерные плоскости $D x_{1} x_{2}$ и $D x_{3} x_{4}-$ плоскости динамической симметрии тела.

Неконсервативная сила (сопротивления) $\mathbf{S}=\left\{S_{1}, S_{2}, 0,0\right\}$ и координаты точки ее приложения $N=\left(0,0, x_{3 N}, x_{4 N}\right)$ в системе $D x_{1} x_{2} x_{3} x_{4}$ вместе с соотношениями $S_{1}=$ $\sigma \sin \gamma, S_{1}=-S \cos \gamma, \gamma=$ const, $x_{3 N}=R \cos \beta_{1}, x_{4 N}=R \sin \beta_{1}(\gamma-$ угол в плоскости $D x_{1} x_{2}$ и $\beta_{1}-$ угол в плоскости $\left.D x_{3} x_{4}\right)$ позволяют получить динамическую часть уравнений движения на $\mathbb{R}^{4} \times s o(4)$.

Если прямая $C D\left(C\right.$ - центр масс) лежит в плоскости $D x_{1} x_{2}$ и вектор DC определяет положение центра масс: $\mathbf{D C}=\{\sigma \sin \gamma,-\sigma \cos \gamma, 0,0\}$, то вектор скорости $\mathbf{v}_{D}$ точки $D$ можно представить в следующем виде:

$$
\mathbf{v}_{D}=\left\{v \cos \alpha \sin \beta_{2}, v \cos \alpha \cos \beta_{2}, v \sin \alpha \cos \beta_{1}, v \sin \alpha \sin \beta_{1}\right\}, \quad\left|\mathbf{v}_{D}\right|=v
$$

$\left(\beta_{2}-\right.$ угол в плоскости $\left.D x_{1} x_{2}\right)$.

Пусть $\Omega$ - тензор угловой скорости тела, $\Omega \in \operatorname{so}(4)$. Та часть уравнений движения, которая отвечает алгебре $\operatorname{so}(4)$, имеет вид [4]-[6]: $\dot{\Omega} \Lambda+\Lambda \dot{\Omega}+[\Omega, \Omega \Lambda+\Lambda \Omega]=M$, где $\Lambda=\operatorname{diag}\left\{\lambda_{1}, \lambda_{2}, \lambda_{3}, \lambda_{4}\right\}, \lambda_{1}=\left(-I_{1}+I_{2}+I_{3}+I_{4}\right) / 2, \ldots, \lambda_{4}=\left(I_{1}+I_{2}+I_{3}-I_{4}\right) / 2$, a $M-$ момент внешних сил, действующих на тело в $\mathbb{R}^{4}$, спроектированный на $\operatorname{so}(4),[\cdot]$ коммутатор в so(4). Кососимметрическую матрицу $\Omega \in \operatorname{so}(4)$ удобно представлять в следующих естественных координатах:

$$
\left(\begin{array}{cccc}
0 & -\omega_{6} & \omega_{5} & -\omega_{3} \\
\omega_{6} & 0 & -\omega_{4} & \omega_{2} \\
-\omega_{5} & \omega_{4} & 0 & -\omega_{1} \\
\omega_{3} & -\omega_{2} & \omega_{1} & 0
\end{array}\right)
$$

где $\omega_{1}, \omega_{2}, \omega_{3}, \omega_{4}, \omega_{5}, \omega_{6}$ - компоненты угловой скорости в проекциях на алгебру so(4).

При вычислении момента внешней силы необходимо построить отображение $\mathbb{R}^{4} \times$ $\mathbb{R}^{4} \longrightarrow \mathrm{so}(4)$, переводящее пару векторов из $\mathbb{R}^{4}$ в некоторый элемент из алгебры so(4).

Очевидно, что имеются два циклических интеграла: $\omega_{1}=\omega_{1}^{0}=$ const, $\omega_{6}=\omega_{6}^{0}=$ const. Будем рассматривать динамику системы на нулевых их уровнях: $\omega_{1}^{0}=\omega_{6}^{0}=0$.

Для получения явного вида динамических уравнений определим две функции воздействия среды $R$ и $S$ (используя при этом аналогичную информацию о свойствах движения трехмерных тел [3], [7]): $R=R(\alpha)=A \sin \alpha, S=S_{v}(\alpha)=B v^{2} \cos \alpha$; $A, B>0$. Тогда редуцированные уравнения перепишутся как $\dot{\omega}_{2}=-n_{0}^{2} v^{2} \sin \alpha \cos \alpha \times$ $\sin \beta_{1} \cos \gamma, \dot{\omega}_{3}=-n_{0}^{2} v^{2} \sin \alpha \cos \alpha \sin \beta_{1} \sin \gamma, \dot{\omega}_{4}=n_{0}^{2} v^{2} \sin \alpha \cos \alpha \cos \beta_{1} \cos \gamma, \dot{\omega}_{5}=$ $n_{0}^{2} v^{2} \sin \alpha \cos \alpha \cos \beta_{1} \sin \gamma\left(n_{0}^{2}=A B /\left(I_{1}+I_{3}\right)\right)$.

Несколько расширим задачу. Предположим, что в плоскости $D x_{1} x_{2}$ действует следящая сила $\mathbf{T}$, линия действия которой проходит через центр масс $C$. При этом рассмотрим класс движений, при котором во все моменты времени выполнены условия (неинтегрируемые связи): $v=$ const, $\beta_{2}=$ const [3].

Работа выполнена при поддержке РФФИ (грант № 08-01-00231-а). 
В результате замены угловых скоростей по формулам $z_{1}=\omega_{3} \cos \beta_{1}+\omega_{5} \sin \beta_{1}$, $z_{2}=\omega_{3} \sin \beta_{1}-\omega_{5} \cos \beta_{1}, z_{3}=\omega_{2} \cos \beta_{1}+\omega_{4} \sin \beta_{1}, z_{4}=\omega_{2} \sin \beta_{1}-\omega_{4} \cos \beta_{1}, w_{1}=$ $-z_{1} \sin \beta_{2}+z_{3} \cos \beta_{2}, w_{2}=z_{3} \sin \beta_{2}+z_{1} \cos \beta_{2}, w_{3}=z_{2} \sin \beta_{2}-z_{4} \cos \beta_{2}, w_{4}=z_{4} \sin \beta_{2}+$ $z_{2} \cos \beta_{2}$ у редуцированной системы шестого порядка появляется независимая подсистема третьего порядка

$$
\begin{gathered}
\dot{\alpha}=-w_{3}+\sigma n_{0}^{2} v \sin \alpha, \\
\dot{w}_{3}=n_{0}^{2} v^{2} \sin \alpha \cos \alpha \cos \left(\gamma+\beta_{2}\right)-w_{1}^{2} \frac{\cos \alpha}{\sin \alpha}, \\
\dot{w}_{1}=w_{3} w_{1} \frac{\cos \alpha}{\sin \alpha},
\end{gathered}
$$

а также может быть выделена система второго порядка

$$
\dot{w}_{4}=-n_{0}^{2} v^{2} \sin \alpha \cos \alpha \sin \left(\gamma+\beta_{2}\right)+w_{1} w_{2} \frac{\cos \alpha}{\sin \alpha}, \quad \dot{w}_{2}=-w_{4} w_{1} \frac{\cos \alpha}{\sin \alpha}
$$

и уравнение

$$
\dot{\beta}_{1}=w_{1} \frac{\cos \alpha}{\sin \alpha} .
$$

Как было доказано ранее [3], [7], система (1), (3) обладает тремя, вообще говоря, независимыми первыми интегралами следующего вида:

$$
\begin{aligned}
& \Phi_{1}\left(w_{1}, w_{3}, \sin \alpha\right)=\left(w_{1} \sin \alpha\right)^{-1} \\
& \quad \times\left(w_{1}^{2}+w_{3}^{2}-\sigma n_{0}^{2} v w_{3} \sin \alpha+n_{0}^{2} v^{2} \cos \left(\gamma+\beta_{2}\right) \sin ^{2} \alpha\right)=C_{1}=\mathrm{const}, \\
& \Phi_{2}\left(w_{1}, w_{3}, \sin \alpha\right)=C_{2}=\mathrm{const}, \\
& \Phi_{3}\left(w_{1}, w_{3}, \sin \alpha, \beta_{1}\right)=C_{3}=\mathrm{const} .
\end{aligned}
$$

Все три указанных первых интеграла являются трансцендентными функциями своих фазовых переменных, выражающимися через элементарные функции [3], [8].

В силу указанных редукций в рассматриваемой системе шестого порядка для полного ее интегрирования достаточно указать еще один первый интеграл, независимый с интегралами (4)-(6).

После замены переменных

$$
w_{*}=w_{3} \sin \left(\gamma+\beta_{2}\right)+w_{4} \cos \left(\gamma+\beta_{2}\right), \quad w_{* *}=w_{1} \sin \left(\gamma+\beta_{2}\right)-w_{2} \cos \left(\gamma+\beta_{2}\right)
$$

система (2) может быть приведена к виду $d w_{*} / d \beta_{1}=-w_{* *}, d w_{* *} / d \beta_{1}=w_{*}$, который предполагает наличие аналитического первого интеграла: $w_{*}^{2}+w_{* *}^{2}=C_{4}=$ const.

Итак, исследуемая динамическая система вполне интегрируема в классе, вообе говоря, трансцендентных функций (имеющих существенно особые точки).

\section{Список литературы}

[1] М. В. Шамолин, Докл. РАН, 375:3 (2000), 343-346. [2] Д. В. Георгиевский, М. В. Шамолин, Докл. РАН, 380:1 (2001), 47-50. [3] М. В. Шамолин, Методы анализа динамических систем с переменной диссипацией в динамике твердого тела, Изд-во "Экзамен”, М., 2007. [4] Б. А. Дубровин, С. П. Новиков, А. Т. Фоменко, Современная геометрия. Методъ и приложения, Наука, М., 1979. [5] В. В. Трофимов, Вестн. Моск. ун-та. Сер. 1. Матем., мех., 1984, №6, 31-33. [6] О. И. Богоявленский, Докл. АН СССР, 287:5 (1986), 1105-1108. [7] М. В. Шамолин, Докл. РАН, 364:5 (1999), 627-629. [8] М. В. Шамолин, УМН, 53:3 (1998), 209-210.

M. В. Шамолин (M. V. Shamolin)

Институт механики МГУ им. М. В. Ломоносова

E-mail: shamolin@imec.msu.ru
Представлено А. В. Михалёвым Принято редколлегией 09.08.2009 